\title{
Naringenin reduces lung metastasis in a breast cancer resection model
}

\author{
Lei Qin ${ }^{1,2}$, Lingtao Jin ${ }^{1}$, Linlin Lu ${ }^{1}$, Xiaoyan $\mathrm{Lu}^{3}$, Chunling Zhang ${ }^{1}$, Fayun Zhang ${ }^{1}$, Wei Liang ${ }^{1 凶}$ \\ ${ }^{1}$ Protein \& Peptide Pharmaceutical Laboratory, National Laboratory of Biomacromolecules, Institute of Biophysics, Chinese \\ Academy of Sciences, Beijing 100101, China \\ ${ }^{2}$ Graduate University of Chinese Academy of Sciences, Beijing 100049, China \\ ${ }^{3}$ Pharmaceutical Informatics Institute, College of Pharmaceutical Sciences, Zhejiang University, Hangzhou 310058, China \\ 凶 Correspondence: weixx@sun5.ibp.ac.cn \\ Received April 1, 2011 Accepted May 16, 2011
}

\begin{abstract}
Metastasis is the main cause of death in cancer patients. To improve the outcomes of patients undergoing a surgery, new adjuvant therapies that can effectively inhibit metastases have to be developed. Studies have shown that flavonoid naringenin, a natural product that is mainly present in grapes and citrus, may contribute to cancer prevention. It has many advantages compared to traditional chemotherapeutic drugs, such as low toxicity. To determine whether naringenin can also inhibit metastases, a breast cancer resection model that mimics clinical situations was established. We found that orally administered naringenin significantly decreased the number of metastatic tumor cells in the lung and extended the life span of tumor resected mice. Flow cytometry analysis revealed that $T$ cells displayed enhanced antitumor activity in naringenin treated mice, with an increased proportion of IFN- $\mathrm{Y}$ and IL-2 expressing T cells. In vitro studies further demonstrated that relief of immunosuppression caused by regulatory $T$ cells might be the fundamental mechanism of metastasis inhibition by naringenin. These results indicate that orally administered naringenin can inhibit the outgrowth of metastases after surgery via regulating host immunity. Thus, naringenin can be an ideal surgical adjuvant therapy for breast cancer patients.
\end{abstract}

KEYWORDS naringenin, breast cancer, surgery, metastasis, immunosuppression, Tregs

\section{INTRODUCTION}

Breast cancer is the most common cancer in women and the second leading cause of cancer death among women worldwide (Coughlin and Ekwueme, 2009; Jemal et al., 2010). For most patients, the best treatment is surgery (McCahill et al., 2009); depending on the characteristics of the tumor and the condition of the patients, it is often combined with chemotherapy, and sometimes with radiation therapy, endocrine therapy, or immunotherapy. The main problem of surgery is the likelihood of tumor metastasis and local relapse. Most patients have already developed metastases when their cancer is diagnosed, even if the metastases are not clinically detectable. Although chemotherapy may help reduce the risk of metastasis and relapse, severe side effects on normal cells, especially immune cells, limit its long-term usage. In addition, a recent study has shown that surgery promotes tumor cell dissemination and metastasis (van der Bij et al., 2008). For these reasons, there is an urgent need to develop new adjuvant therapies that have low toxicity and high efficiency in inhibiting the outgrowth of metastases.

The immune system has been shown to be extremely important in struggling against cancers and maintaining host immune surveillance (Bindea et al., 2010). Immune cells can kill tumor cells and thus inhibit tumor growth and metastasis, or even cause a primary tumor rejection in immunogenic tumor models (Townsend and Allison, 1993). However, in patients and poorly immunogenic tumor models, most tumors are not rejected. Mechanisms that underlie T cell tolerance or anergy include negative regulation of the host immune system (e.g. regulatory $\mathrm{T}$ cells [Tregs], myeloid-derived suppressor cells [MDSC]), immunosuppression induced by tumor-derived molecules, and lack of effective recognition of tumors by T cells (e.g. the absence of T-cell antigen receptor [TCR] and/or costimulatory signals). Of the above factors, Tregs play a critical role in suppressing antitumor immune responses. They inhibit $\mathrm{T}$ cell activation and proliferation by 
producing immunosuppressive cytokines transforming growth factor- $\beta$ (TGF- $\beta$ ) and IL-10. Tregs are apparently elevated in cancer patients, and high amounts of Treg cells are closely correlated with poor prognosis (Liyanage et al., 2002; Beyer and Schultze, 2006). In addition, surgery itself causes a generalized state of immunosuppression due to tissue damage, anesthetic and analgesic drugs, hypothermia, blood loss, transfusion, pain and perioperative distress, etc. (Ben-Eliyahu, 2003; Boomsma et al., 2010; Hogan et al., 2011). The critical factor for successful treatment of tumor resection patients is the elimination of residual tumor cells. Immune cells are distributed all over the body and have a high likelihood to contact tumor cells. Therefore, relieving immunosuppression and restoring systemic antitumor immunity can improve the outcomes of patients undergoing tumor surgery.

4T1 is a mouse mammary carcinoma cell line with many similarities to human breast cancer, which makes it a suitable animal model for investigation of human breast cancer. 4T1 cells are highly malignant and quickly develop spontaneous metastases to various organs, with pulmonary metastasis as the most common and predominant cause of death (Chen et al., 2007). 4T1 tumors are lethal even after the primary tumor has been removed (duPre et al., 2008). Furthermore, 4T1 tumors are poorly immunogenic, and resist many immunebased therapies. Thus, it has become a very challenging model to evaluate immunotherapy (Chen et al., 2007).

Naringenin, a flavonoid that is present in high concentrations in grapefruits and citrus fruits, is considered to be a safe natural product with a wide spectrum of pharmacological activities. Previous studies have indicated that naringenin has anti-cancer effects; however most studies focused on its role in primary tumor prevention (So et al., 1996; Le Marchand et al., 2000; Kanno et al., 2005), and few investigated its antimetastatic effects. We previously found that naringenin could attenuate bleomycin-induced pulmonary fibrosis by improving the immunosuppressive environment ( $\mathrm{Du}$ et al., 2009). Furthermore, relief of pulmonary fibrosis by naringenin led to a decrease in lung metastasis when challenged with subcutaneously injected or intravenously injected tumor cells. However, it remains unknown whether naringenin may still inhibit metastases in mice without pulmonary fibrosis, especially in the surgery models that closely resemble clinical situations. We hypothesized that immunosuppression might be the reason for metastatic cancer cell survival and the final surgical failure of $4 \mathrm{~T} 1$ tumor-bearing mice. As naringenin could modulate the immune system of mice with pulmonary fibrosis, it might help the host to fight against metastatic cancer cells and inhibit metastases.

In the present study, we examined the anti-metastatic effects of orally administered naringenin using a highly malignant 4T1 murine breast cancer model and investigated whether naringenin could inhibit metastases in a surgical resection model, and discuss possible mechanisms.

\section{RESULTS}

\section{Naringenin inhibits lung metastasis and extends survival of mice in a breast cancer resection model}

An orthotopic 4T1 breast cancer resection model was established to mimic clinical situations (Fig. 1A). Primary tumors were resected on day 14 , when spontaneous metastasis already occurred (Pulaski and OstrandRosenberg, 2001). Since day 11, a daily dose of $100 \mathrm{mg} / \mathrm{kg}$ naringenin was administered orally for 24 days. Efficacy of naringenin was evaluated by the lung clonogenic metastasis assay and the long-term survival experiment. The results showed that the naringenin treatment group $(100 \mathrm{mg} / \mathrm{kg}$ ) exhibited significantly decreased number of lung metastatic colonies compared to the nontreatment surgical control group on both day 3 (13-fold) and day 7 (6-fold) after resection, suggesting that naringenin efficiently inhibits lung metastases $(P<0.01$; Fig. 1B). Moreover, as shown in Fig. S1, mice that underwent surgical resection showed a $\sim 2.5$-fold higher number of metastatic tumor cells than the nonsurgical mice, indicating that surgery promotes tumor metastases.

We further examined the long-term benefit of naringenin. The naringenin treatment group showed a prolonged survival compared to the nontreatment surgical control group (median survival, 74.5 days vs 53.5 days, $p<0.05$; Fig. $1 \mathrm{C}$ ). At the end of the experiment (on day 160), 3 of $10(30 \%)$ mice in the treatment group were still alive and tumor-free, while all mice in the nontreatment group died by day 104. Taken together, these results indicate that orally administered naringenin can inhibit tumor metastases to the lung and prolong the life span of tumor resected mice.

\section{Naringenin does not inhibit tumor cell proliferation either in vivo or in vitro}

In order to find whether the antimetastatic effect of naringenin is due to the inhibition of metastatic tumor cell proliferation, we examined the inhibitory effect of naringenin on 4T1 cancer cell proliferation in vitro using the MTT assay, and in vivo by orthotopic 4T1 tumor growth measurement. Results showed that naringenin did not obviously inhibit tumor proliferation either in vitro or in vivo (Fig. $2 \mathrm{~A}$ and $2 \mathrm{~B}$ ).

In the MTT assay, naringenin did not have obvious effect on the cell viability of $4 \mathrm{~T} 1$ tumor cells, but slightly enhanced cell proliferation at concentrations ranging from 6.25 to $100 \mu \mathrm{mol} / \mathrm{L}$ (Fig. 2A). The in vivo assay showed that naringenin did not inhibit tumor growth (Fig. 2B). In addition, in the tumor resection model, there was no significant difference in tumor volume between the naringenin treated mice and the untreated mice at the time of surgery, although naringenin had been given for 3 days (data not shown). These results suggest that naringenin does not kill tumor cells directly. 


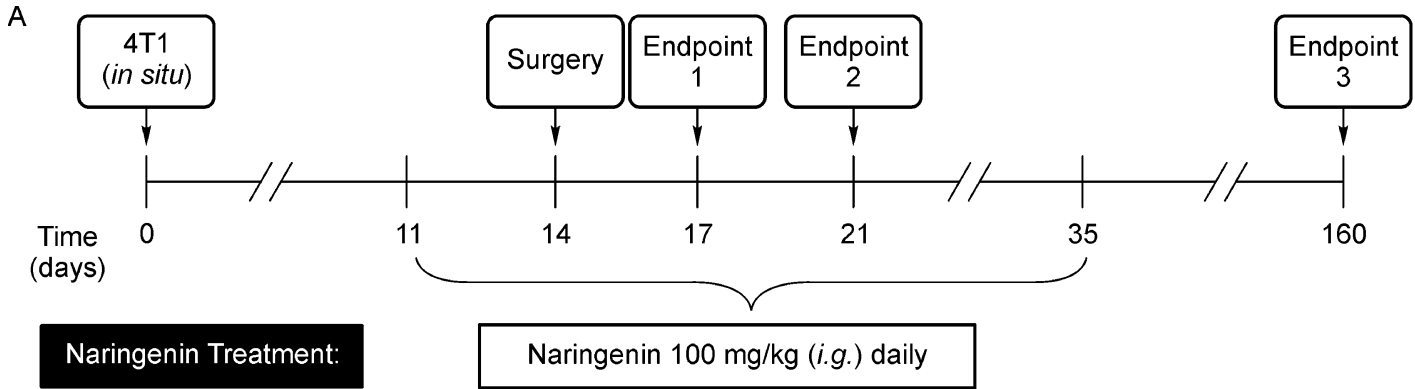

B

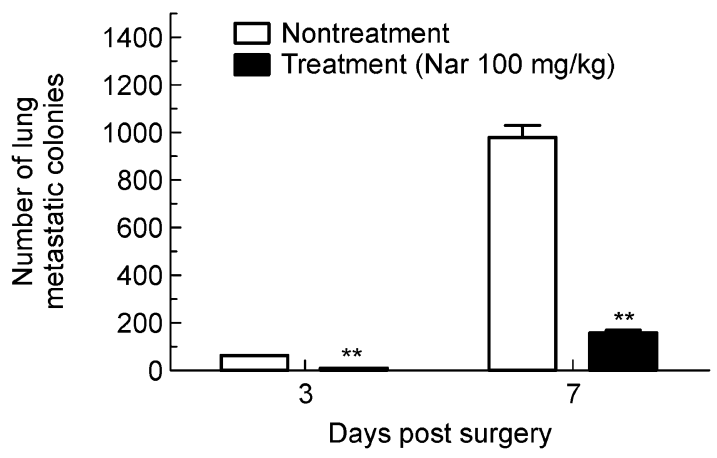

C

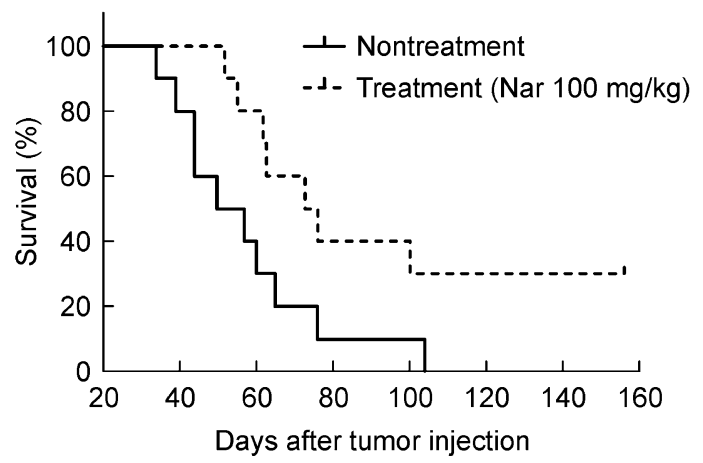

Figure 1. Efficacy of naringenin in a tumor resection model. (A) The schematic diagram of the animal experiment. Briefly, $2.0 \times 10^{5} 4 \mathrm{~T} 1$ cancer cells were injected into the fourth mammary fat-pad of female BALB/c mice on day 0 . The primary tumor was resected on day 14 . Since day 11 , mice were given a daily dose of $100 \mathrm{mg} / \mathrm{kg}$ naringenin (treatment group) or vehicle (nontreatment group), and the treatment was continued for 24 days. (B) Lung metastases were determined by clonogenic metastasis assay 3 and 7 days after surgery (on days 17 and 21). Data are presented as mean $\pm \mathrm{SD} ; n=3$ mice/group. ${ }^{* *} p<0.01$ for treatment group versus the nontreatment group at corresponding time points (Student's $t$-test). (C) The life span of tumor resected mice that were treated with either naringenin or vehicle was evaluated within 160 days. $n=10$ mice/group. $p<0.05$ for treatment group versus nontreatment group (log-rank test).

A

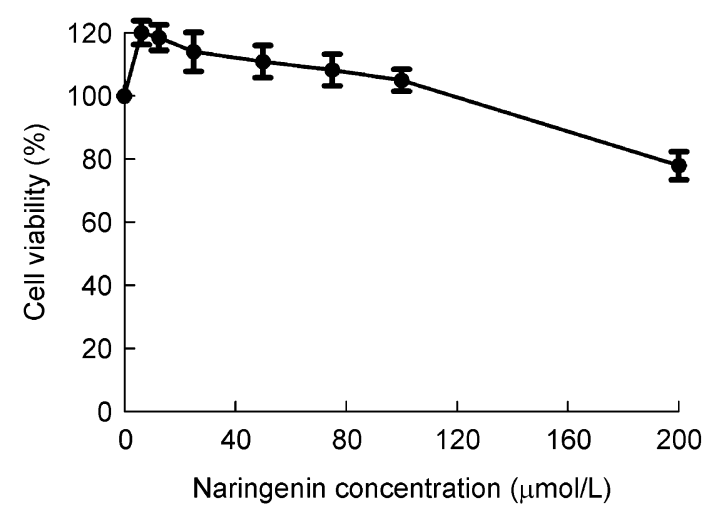

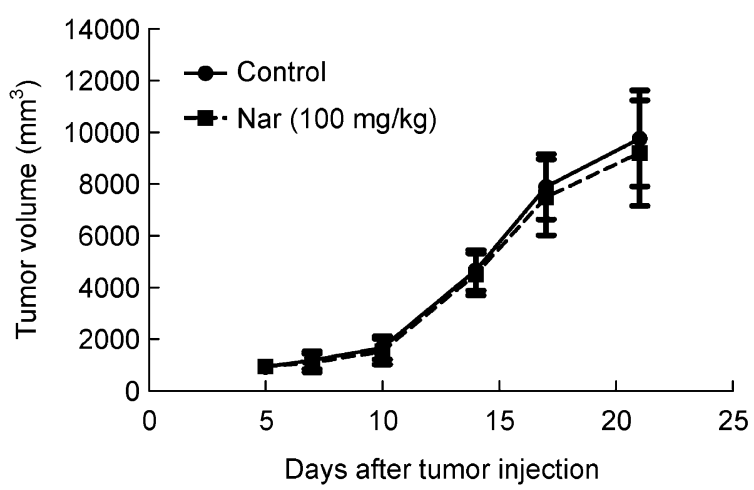

Figure 2. Inhibitory effect of naringenin on $4 \mathrm{~T} 1$ cell growth. (A) The viability of naringenin treated $4 \mathrm{~T} 1$ cells was determined by MTT assay. Cells were treated for $24 \mathrm{~h}$ and the cell viability was calculated by absorbance of treated cells relative to that of untreated cells. Data are presented as mean $\pm S D$. (B) The in vivo growth inhibitory effect of naringenin was examined by primary tumor growth. Mice received a mammary fat-pad injection of $2.0 \times 10^{5} 4 \mathrm{~T} 1$ cancer cells on day 0 . Starting from the following day, naringenin was administered for two weeks at $100 \mathrm{mg} / \mathrm{kg}$ (i.g.). Data are presented as mean $\pm \mathrm{SD} ; n=10$ mice/group. 


\section{Naringenin promotes $\mathrm{T}$ cell activation and restores $\mathrm{T}$ cell function in mice}

The antitumor immune function is mainly mediated through $\mathrm{T}$ cells, especially $\mathrm{CD}^{+}$cytotoxic $\mathrm{T}$ lymphocytes (CTL) (Rosenberg, 2001). However, in cancer patients, the antitumor function of immune system is often impaired, which leads to uncontrolled tumor growth (Brandacher et al., 2006). Furthermore, surgery itself causes severe immunosuppression, and impaired $T$ cell function after surgery has been frequently observed in several studies (Brune et al., 1999; Ogawa et al., 2000). Thus, we hypothesized that the antimetastatic effect of naringenin was probably due to the recovery of $T$ cell function. To prove this hypothesis, we examined several $T$ cell surface receptors and activation markers, and found that naringenin increased the proportion of activated T cells (CD44 ${ }^{\text {high }} \mathrm{CD} \mathrm{L}^{-}$subpopulation) in lung $\mathrm{CD}^{+}$(from $28.4 \%$ to $37.3 \%, p<0.01$ ) and $\mathrm{CD} 8^{+} \mathrm{T}$ cells (from $30.8 \%$ to $39.4 \%, p<0.05$ ) (Fig. $3 \mathrm{~A}$ ). These results suggest that naringenin can promote $\mathrm{T}$ cell activation.

We also evaluated the antitumor activity of $\mathrm{CD}^{+} \mathrm{T}$ lymphocytes through their ability to express interferon- $\gamma$ (IFN- $\gamma$ ) and IL-2. IFN- $\gamma$ is regarded to be critical for tumor eradication (Ko et al., 2005). We found that the number of IFN-y expressing splenic $\mathrm{CD} 8^{+} \mathrm{T}$ cells sharply decreased in the tumor-bearing mice undergoing surgery compared to the normal control mice (from $6.8 \%$ to $2.2 \%$ ). However, naringenin treatment significantly restored the IFN-y expressing $\mathrm{T}$ cells to normal level (from 2.2\% to $7.8 \%, p<0.05$ ) (Fig. 3B and $3 \mathrm{C})$. In addition, IL-2 expressing CD $8^{+}$T cells also elevated to normal levels in the naringenin treated mice (from 5.6\% to $12.1 \%, p<0.01$; Fig. 3B and 3D). Taken together, these results indicate that naringenin can promote $\mathrm{T}$ cell activation and restore the antitumor function of T cells in tumor resected mice.

\section{Naringenin downregulates the expression of immunosuppressive cytokines in T cells in vitro}

We further investigated the possible mechanisms for the recovery of $\mathrm{T}$ cell function in vitro. Immune suppression is often observed in animal tumor models and cancer patients, and it contributes to the failure of immune therapies (Whiteside, 2006). Furthermore, surgery causes a Treg predominant environment, which further intensifies immunosuppression in patients (Hogan et al., 2011). We hypothesized that the recovery of antitumor function by naringenin was due to the relief of immunosuppression. To assess this possibility, we examined the production of immune suppressive cytokines TGF- $\beta 1$ and IL- 10 by naive splenic T cells using ELISA and flow cytometry methods. We found that naringenin significantly decreased the level of secreted TGF- $\beta 1$ and the numbers of $\mathrm{CD}^{+}$and $\mathrm{CD} 8^{+}$cells to less than $50 \%$ of control values $(p<0.01$; Fig. $4 \mathrm{~A})$. Flow cytometric assay also revealed a significant reduction in IL-10 expressing T cells $(P<0.01$; Fig. 4B). These results indicate that naringenin can improve TGF- $\beta 1$ and IL-10 mediated immunosuppression.

\section{Naringenin inhibits the production of Tregs in vitro}

TGF- $\beta 1$ and IL-10 are immunosuppressive cytokines that are mainly produced by a subpopulation of $T$ cells called Tregs, an important type of immunosuppressive cells. Tumorderived factors can mediate the conversion of naive T cells into Tregs. To determine whether naringenin inhibits the conversion of naive T cells into Tregs, we used naringenin to treat naive T cells. While TGF- $\beta 1$ significantly induced the conversion of naive Tcells to $\mathrm{CD} 4^{+} \mathrm{CD} 25^{+}{ }^{+}$oxp $3^{+}$Tregs (from $4.5 \%$ to $23.7 \%$ ), naringenin significantly reversed this process (from $23.7 \%$ to $10.7 \%$ ) (Fig. 5A and 5B). These data suggest that naringenin might inhibit Treg production, which, in turn, recovers $T$ cell function.

\section{DISCUSSION}

Chemotherapy drugs are commonly used to inhibit metastases in breast cancer patients who undergo surgery (Markiewicz et al., 1996; Shenkier et al., 2004). However, the severe side effects (such as immunosuppression and myelosuppression) limit the long-term usage of chemotherapy. Naringenin is a natural dietary supplement and has a clear safety record. The medium lethal dose (LD50) of naringenin is $>5000 \mathrm{mg} / \mathrm{kg}$ in mice and rats (Ortiz-Andrade et al., 2008). In the present study, we examined the antimetastasis effect of naringenin in a breast cancer resection model. Results showed that orally administered naringenin significantly decreased the number of metastatic tumor cells in the lung (Fig. 1B) and extended the life span of tumor resected mice (Fig. 1C), which makes it a promising adjuvant drug for tumor resection patients.

The in vivo experiments were performed using a most challenging $4 \mathrm{~T} 1$ model, which is highly malignant and poorly immunogenic. Long-term survival is seldom observed in this model (Lohr et al., 2000; Pulaski et al., 2000). However, we found a prolonged survival in naringenin treated mice, among which $30 \%$ showed long-term survival (Fig. 1C). This is attributed to the inhibition of lung metastasis by naringenin (Fig. 1B). Because naringenin does not directly inhibit tumor proliferation (Fig. 2A and 2B), its antimetastatic effect may be due to other mechanisms. In addition, we found that surgery itself can promote metastases to lungs (Fig. S1), which is consistent with other reports (Page and Ben-Eliyahu, 1997; van der Bij et al., 2008).

The tumor resection model has its unique characteristics. Surgery may cause tumor cells to detach from primary tumors and disseminate throughout the body (van der Bij et al., 2008). Meanwhile, cell-mediated immunity is suppressed after surgery (Faist et al., 1986; Salo, 1992; Boomsma et al., 

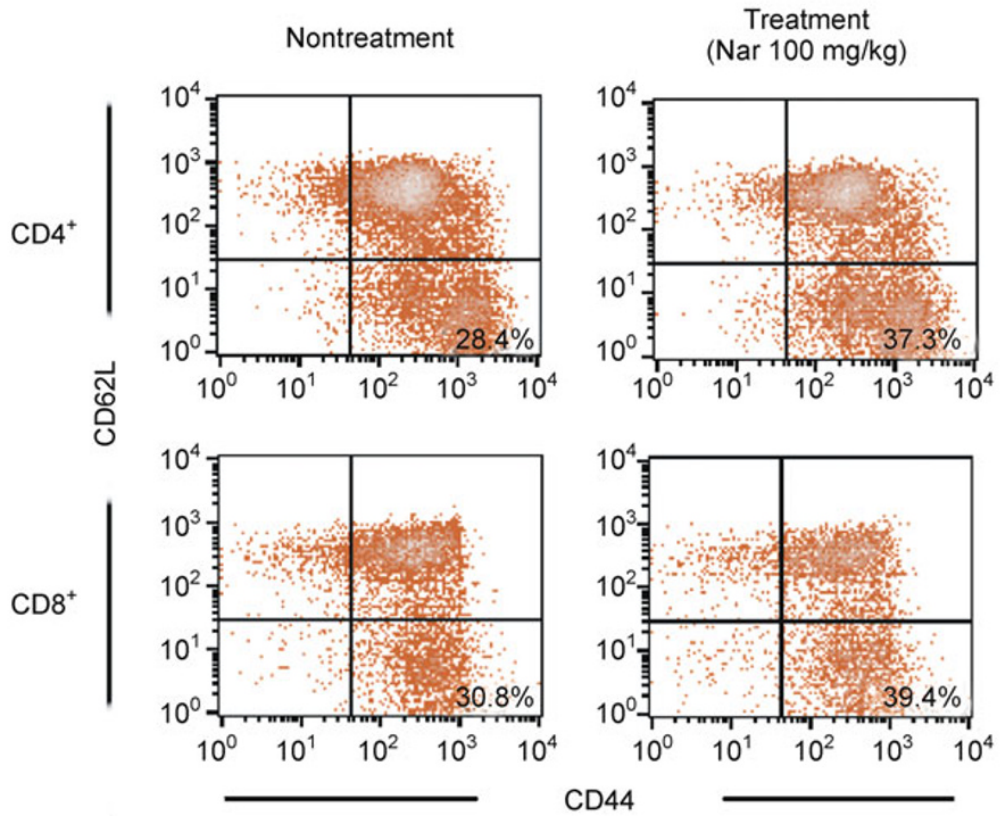

B
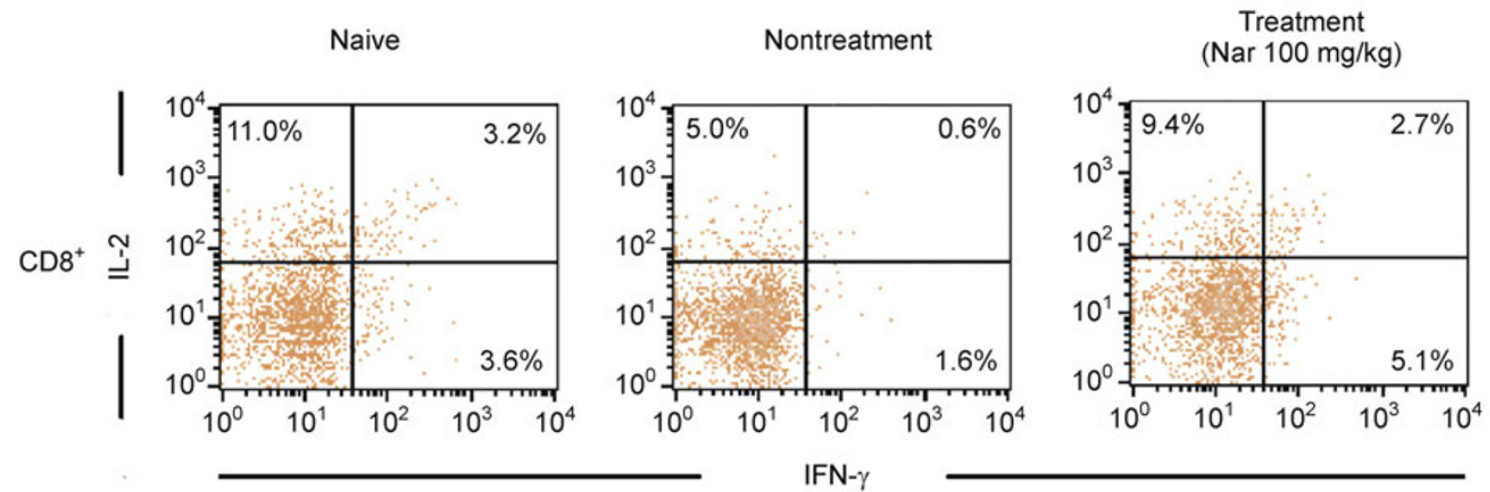

C

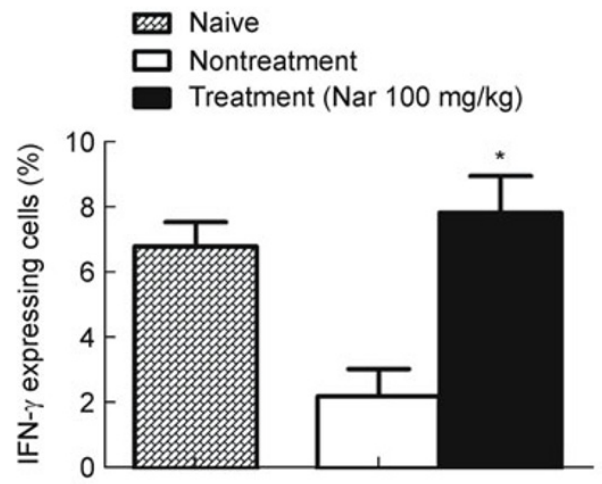

D

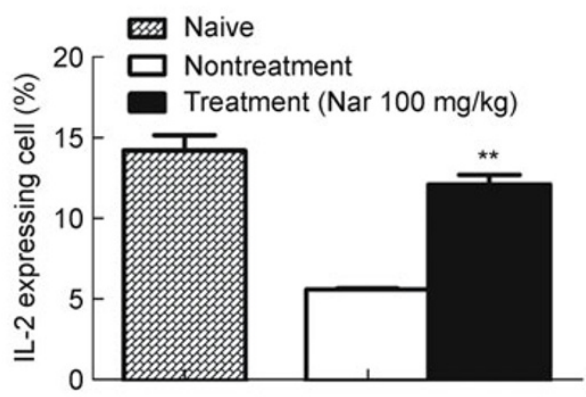

Figure 3. Naringenin promotes $\mathbf{T}$ cell activation and restores $\mathbf{T}$ cell function in a tumor resection model. Naringenin treated mice, untreated mice or normal naive mice were killed 7 days after surgery. T cells from lungs and spleens were analyzed for expression of cell surface markers and intracellular cytokines using flow cytometry. (A) Naringenin treated mice displayed elevated proportion of activated T cells (CD44 ${ }^{\text {high }} \mathrm{CD}_{2} \mathrm{~L}^{-}$) in $\mathrm{CD}^{+}$(upper) and $\mathrm{CD}^{+}$(lower) T cells from lungs. $n=3$ mice/group. (B) Naringenin increased the proportion of IFN- $y$ producing $\mathrm{CD}^{+} \mathrm{T}$ cells and IL-2 producing $\mathrm{CD} 8^{+} \mathrm{T}$ cells. The representative bar graph summarizing the flow cytometry results of $(\mathrm{B})$ is shown in $(C)$ and $(D)$, respectively. Data are given as mean $\pm S D ; n=3$ mice/group. ${ }^{*} p<0.05,{ }^{* *} p<0.01$ for treatment group versus the nontreatment control (Student's $t$-test). 
A

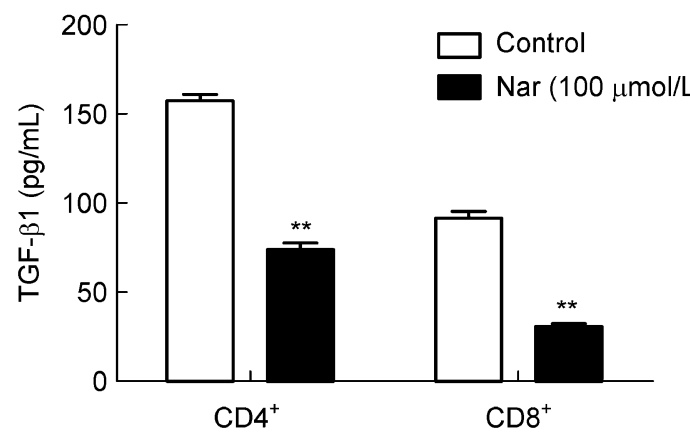

B

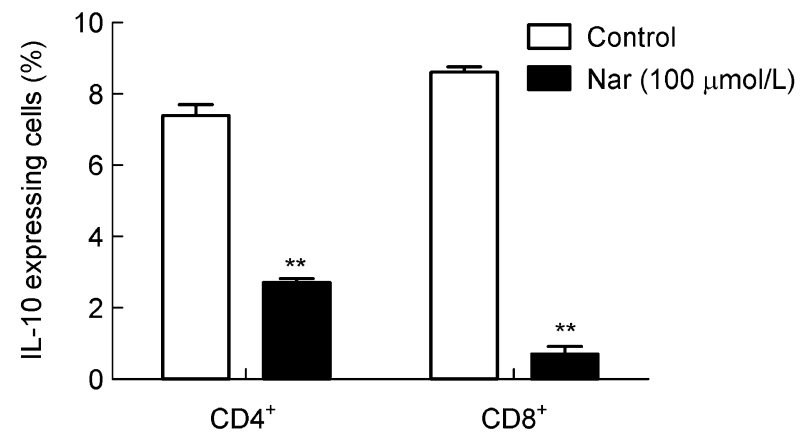

Figure 4. Naringenin treatment downregulates the expression of immunosuppressive cytokines in T cells in vitro. The purified splenic T cells or total splenocytes were cultured with or without naringenin $(100 \mu \mathrm{mol} / \mathrm{L})$ for $72 \mathrm{~h}$ in the presence of anti-CD3 $(3 \mu \mathrm{g} / \mathrm{mL})$ and anti-CD28 $(1 \mu \mathrm{g} / \mathrm{mL})$ antibodies. (A) The TGF- $\beta 1$ protein level in the supernatant (X-VIVO 15 serum-free medium) of purified T cells was determined by ELISA. (B) The intracellular expression of IL-10 in splenic T cells was assessed using flow cytometry. Data are presented as mean $\pm \mathrm{SD} ;{ }^{* *} p<0.01$ for naringenin treatment versus control (Student's $t$-test).

2010), when impaired T cell function and Treg predominance have been observed in many studies (Shafir et al., 1980; Brune et al., 1999; Ogawa et al., 2000; Hogan et al., 2011). The detached tumor cells and the less efficient immune surveillance may account for the metastasis-promoting effect of surgery. Since the disseminated tumor cells have a great chance of encountering immune cells that are distributed throughout the entire body, restoration of the immune cell function is extremely important for eliminating these tumor cells. Therefore, we examined $T$ cell function of the tumor resected mice, especially in spleen, which could reflect the immune states of the whole body. Flow cytometry analysis showed that naringenin treated mice exhibited an increased proportion of IFN-y and IL-2 expressing $\mathrm{CD}^{+} \mathrm{T}$ cells in spleen, and an elevated level of $T$ cell activation marker $\mathrm{CD} 44{ }^{\text {high }} \mathrm{CD} 6 \mathrm{~L}^{-}$in the lung, suggesting the recovery of T cell function (Fig. 3A-D). Since T cells are critical for antitumor immunity, enhanced $T$ cell function of the host may contribute to the antimetastatic effect of naringenin. This result can explain the low efficacy of naringenin on inhibiting 4T1 proliferation in vitro (Fig. 2A), since no T cell existed in the culture system. However, naringenin treatment also failed to inhibit primary tumor growth (Fig. 2B), which might be due to the extremely large number of tumor cells and a high proliferation rate. Tumor cells could be eliminated by activated T cells only when the tumor cells were present in a very small number. Once the primary tumor was removed, the antitumor function of T cells was restored, suggesting that naringenin is efficient in eliminating a small portion of tumor cells.

Interestingly, a recent study reported that naringenin could suppress picrylchloride-induced contact hypersensitivity by inhibiting the proliferation and activation of T cells, while our previous study demonstrated that naringenin could enhance $\mathrm{T}$ cell function in a bleomycin induced pulmonary fibrosis (similar to the present results) (Du et al., 2009; Fang et al., 2010). The different effects of naringenin on T cells may be caused by different disease models and different experiment systems. The present results confirm that naringenin is an immunomodulator-regulating the suppressed or overactivated $\mathrm{T}$ cells to normal levels.

As immunosuppression is severe in cancer patients undergoing surgery and probably contributes to T cell anergy, we examined the effect of naringenin on immunosuppression related molecules and cells in vitro (Liyanage et al., 2002; Beyer and Schultze, 2006). The results revealed that naringenin significantly inhibited the production of TGF- $\beta 1$ and IL-10 (Fig. 4A and 4B). Furthermore, naringenin inhibited TGF- $\beta 1$ induced Treg production in vitro (Fig. $5 A$ and $5 B$ ). These data demonstrate that naringenin can reverse Treg related immunosuppression, which in turn increase the proportion of activated T cells. TGF- $\beta$ signaling is essential for the transcription of Foxp3, a transcription factor necessary for the production of Tregs (Chen and Konkel, 2010). A recent study showed that naringenin could inhibit TGF- $\beta$ ligandreceptor interaction, thus inhibiting TGF- $\beta$ signal transduction and the downstream gene expression (Yang et al., 2011). Therefore, a possible mechanism is that naringenin inhibits TGF- $\beta$ ligand-receptor interaction, and the suppressed TGF- $\beta$ signaling leads to reduced transcription of Foxp3, making it difficult for naive CD4 T cells to develop into Tregs.

The regulation of the immune system is a complicated process and involves many types of cells, such as MDSCs and tumor-associated macrophages (TAMs) (Marigo et al., 2008; Coffelt et al., 2009; Gabrilovich and Nagaraj, 2009). Whether the inhibition of Tregs by naringenin plays a definitive role in $\mathrm{T}$ cell restoration should be clarified in future studies.

In summary, our results indicate that orally administered naringenin can inhibit the outgrowth of metastases after surgery via regulating host immunity. The antimetastatic properties and low toxicity of naringenin make it an ideal surgical adjuvant therapy for breast cancer. In addition, 


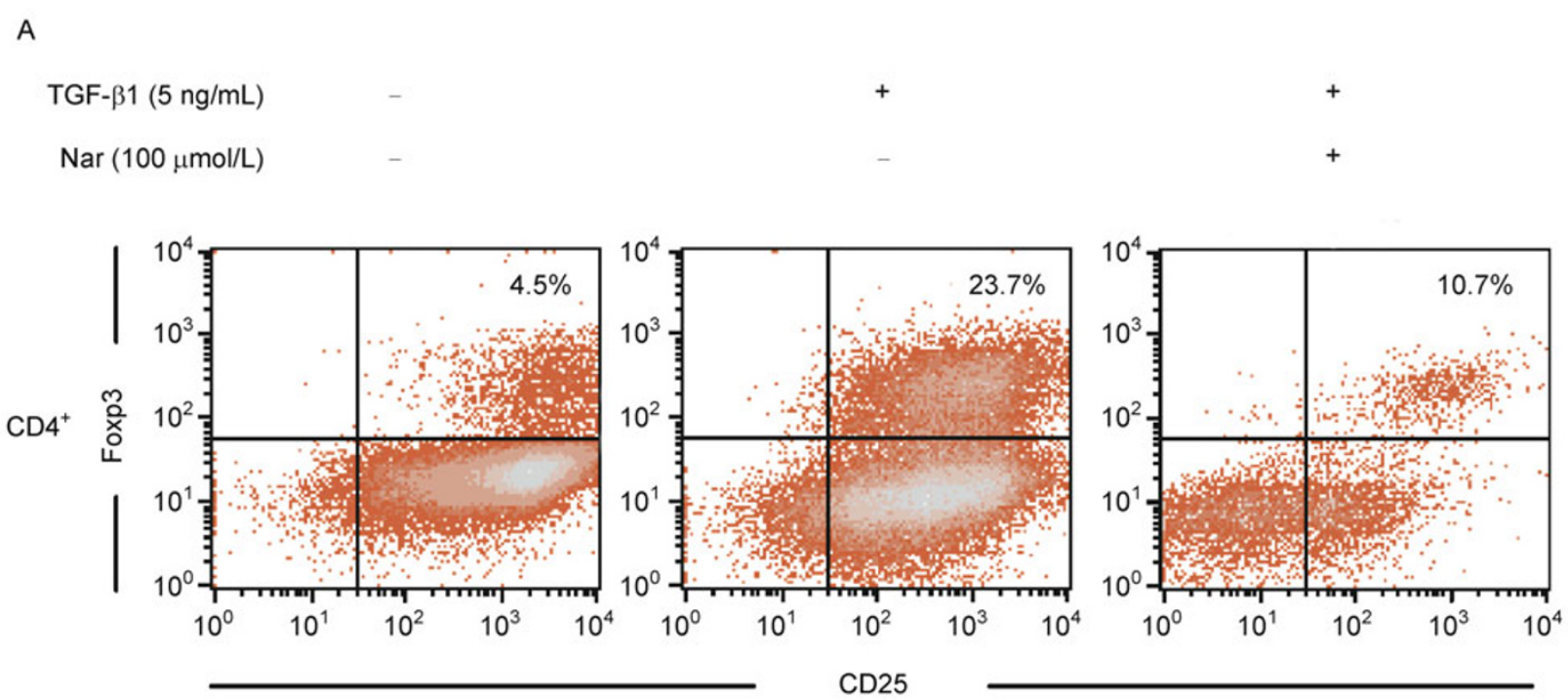

B

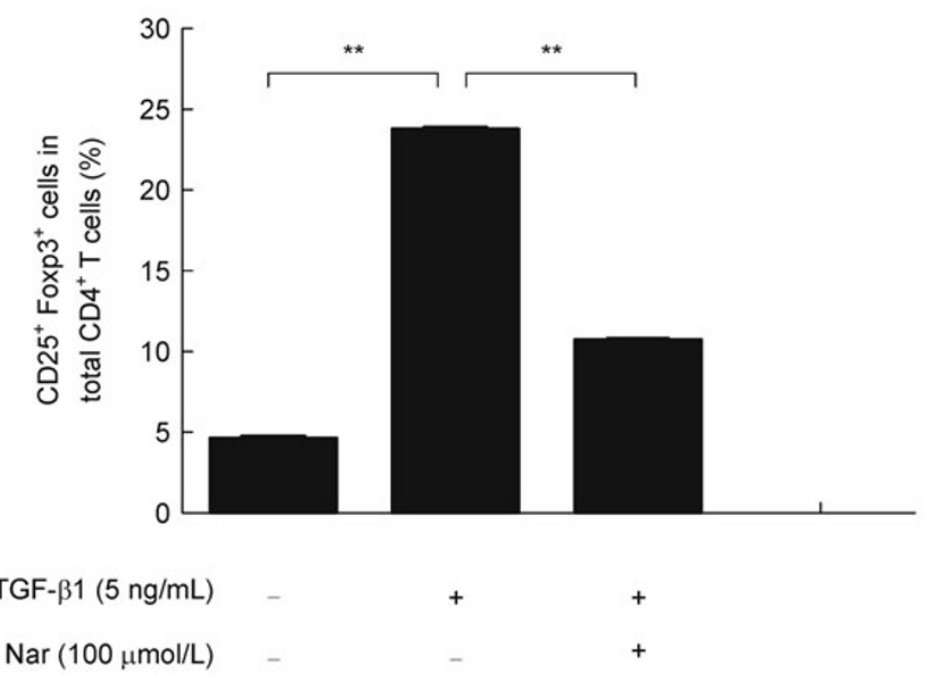

Figure 5. Naringenin inhibits regulatory $\mathrm{T}$ cell production in vitro. Purified splenic CD4 ${ }^{+} \mathrm{T}$ cells were cultured with anti-CD3 antibody $(3 \mu \mathrm{g} / \mathrm{mL})$, anti-CD28 antibody $(1 \mu \mathrm{g} / \mathrm{mL})$ and TGF- $\beta 1(5 \mathrm{ng} / \mathrm{mL})$ to induce regulatory Tcells. Naringenin was supplemented to the culture at concentrations of 0 and $100 \mu \mathrm{mol} / \mathrm{L}$ for $72 \mathrm{~h}$. Cells that were treated with neither TGF- $\beta 1$ nor naringenin were used as normal controls. The proportion of regulatory $\mathrm{T}$ cells $\left(\mathrm{CD} 4^{+} \mathrm{CD} 25^{+} \mathrm{Foxp}^{+}\right)$in total $\mathrm{CD} 4^{+} \mathrm{T}$ cells was analyzed by flow cytometry. Representative flow cytometry data and bar graph summarizing the results are shown in $(A)$ and $(B)$, respectively. Data are presented as mean $\pm \mathrm{SD} ;{ }^{* *} p<0.01$ (Student's $t$-test).

naringenin might be efficient in autoimmune disease treatment for its immunomodulating activities.

\section{MATERIALS AND METHODS}

\section{Animals and reagents}

Six- to eight-week-old female BALB/c mice and C57BL/6 mice were purchased from the Vital River Laboratory Animal Technology Co.
Ltd. and allowed to accommodate to the new environment for at least one week. The mice were kept in a temperature-controlled room with a 12-hour light and dark cycle under specific pathogen-free conditions. All animal procedures were conducted in accordance with guidelines established by the NIH of the USA and the Animal Care and Use Committee of the Institute of Biophysics, CAS, Beijing.

Naringenin was purchased from Shanxi Huike Botanical Development Co. Ltd. Cell culture reagents and type IV collagenase was from Invitrogen. DNase I and 6-thioguanine were obtained from Sigma. 
Recombinant TGF- $\beta 1$ was from PeproTech. A TGF- $\beta 1$ ELISA kit was from Promega. The $T$ cell isolation kit was purchased from Miltenyi Biotec. FITC-conjugated anti-CD4 (GK1.5), Percp-cy5.5-conjugated anti-CD8 (53-6.7), APC-conjugated anti-CD25 (PC-61.5), PE-conjugated anti-CD44 (IM7), APC-conjugated anti-CD62L(MEL-14), purified anti-mouse CD16/32 (93), PE-conjugated anti-IFN-Y (XMG1.2), APC-conjugated anti-IL-2 (JES6-5H4), PE-conjugated anti-IL-10 (JES5-16E3), PE-conjugated anti-Foxp3 (FJK-16s), purified anti-CD3e (145-2C11) and anti-CD28 (37.51) were all purchased from eBioscience.

\section{Cell culture}

4T1 murine mammary cancer cells (6-thioguanine resistant) were obtained from the American Type Culture Collection and cultured in RPMI 1640 supplemented with $10 \%$ heat-inactivated fetal bovine serum, $100 \mathrm{U} / \mathrm{mL}$ penicillin, and $100 \mu \mathrm{g} / \mathrm{mL}$ streptomycin.

\section{Animal models}

To examine the effect of naringenin in a tumor resection model, female BALB/c mice were randomly divided into two groups: the nontreatment surgical control group and naringenin treatment group. Each group received a mammary fat-pad injection of $2.0 \times 10^{5} 4 \mathrm{~T} 1$ cancer cells on day 0 and a tumor resection on day 14 . The naringenin treatment group was administered a daily dose of $100 \mathrm{mg} / \mathrm{kg}$ (i.g.) naringenin (suspended in 1\% CMC-Na) since day 11 , and the nontreatment group received $1 \% \mathrm{CMC}-\mathrm{Na}$ only; the treatment was sustained for 24 days. For survival experiments, the life span of tumor resected mice was observed within 160 days. For lung metastasis determination and other assays, mice were autopsied 3 and 7 days after surgery (on days 17 and 21), lungs and spleens were carefully harvested for the following assessment.

For tumor resection procedures, mice were anesthetized with pentobarbital sodium. An incision was then made around the tumor and the tumor was dissected away from the chest wall. The tumor was then excised with a small margin and the incision was closed with stitches.

To examine the effect of naringenin on primary tumor growth, female BALB/c mice were randomly divided into two groups. Each group received a mammary fat-pad injection of $2.0 \times 10^{5} 4 \mathrm{~T} 1$ cancer cells. On the following day, one group received a daily dose of $100 \mathrm{mg} / \mathrm{kg}$ (i.g.) naringenin (suspended in 1\% CMC-Na), and the other group received $1 \%$ CMC-Na only. The treatment was sustained for two weeks. The primary tumor growth was evaluated by caliper measurement twice a week [volume $=0.5 \times(\text { width })^{2} \times($ length $\left.)\right]$.

\section{Lung clonogenic metastasis assays}

The harvested lungs were rinsed in HBSS to remove the blood. Lungs were minced with scissors and digested at $37^{\circ} \mathrm{C}$ for $90 \mathrm{~min}$ in sterilized HBSS containing $1 \mathrm{mg} / \mathrm{mL}$ type IV collagenase (Invitrogen) and $0.02 \mathrm{mg} / \mathrm{mL}$ DNase I (Sigma) on a platform rocker. The fragments were then filtered through a 70- $\mu \mathrm{m}$ cell strainer (BD Biosciences) and washed twice in HBSS. Cells were then resuspended in the medium containing $60 \mu \mathrm{mol} / \mathrm{L}$ 6-thioguanine (Sigma) and seeded in 6-well tissue culture plates. After 10-14 days, plates were fixed with methanol and stained with $0.03 \%$ methylene blue. The 6-thioguanine resistant colonies were stained and counted. One colony represents one metastatic cancer cell (duPre et al., 2008).

\section{Cell viability assay}

4T1 cells were plated at a density of $7 \times 10^{3}$ cells per well in 96-well plates in complete RPMI 1640 medium and were cultured for $24 \mathrm{~h}$. Cells were then exposed to a series of concentrations of naringenin for $24 \mathrm{~h}$, and the viability of cells was measured using the 3-(4,5dimethylthiazol-2-yl)-2,5-diphenyltetrazolium bromide (MTT) method. Briefly, the culture medium was discarded and $100 \mu \mathrm{L}$ MTT solution $(0.5 \mathrm{mg} / \mathrm{mL}$ in PBS) was added to each well. The plates were incubated at $37^{\circ} \mathrm{C}$ for $4 \mathrm{~h}$. After incubation, the MTT solution was removed and $100 \mu \mathrm{L}$ of DMSO was added to each well for $10 \mathrm{~min}$ at room temperature. Absorbance was recorded at $570 \mathrm{~nm}$ by a plate reader (Thermo, Germany). The percentage of cell viability was calculated by absorbance of treated cells relative to that of untreated cells (James et al., 2009).

\section{Isolation of lymphocytes}

Spleens were harvested from mice and were filtered through $70-\mu \mathrm{m}$ cell strainers to prepare single cell suspensions. Erythrocytes were subsequently removed by lysis using ammonium chloride. After washing, splenocyte preparations were used for subsequent analysis.

Lungs were harvested, rinsed, and then digested at $37^{\circ} \mathrm{C}$ for $90 \mathrm{~min}$ in sterilized HBSS containing $1 \mathrm{mg} / \mathrm{mL}$ type IV collagenase and $0.02 \mathrm{mg} / \mathrm{mL}$ DNase I on a platform rocker. The fragments were then filtered through a $70-\mu \mathrm{m}$ cell strainer and washed twice in HBSS. Cells were subsequently fractioned by centrifugation at 2500 rotation/ min for 20 min on a discontinuous gradient consisting of $70 \%$ and $35 \%$ Percoll solutions (GE Healthcare). The lymphocytes were recovered from the interface and were used for subsequent flow cytometry analysis.

\section{Purification of T cell subpopulation}

Splenocytes from normal naive $\mathrm{C} 57 \mathrm{BL} / 6$ mice were pelleted and resuspended in the MACS buffer (PBS with $0.5 \% \mathrm{BSA}$ and $2 \mathrm{mmol} / \mathrm{L}$ EDTA, pH 7.2) to a final concentration of $1.1 \times 10^{8}$ cells $/ \mathrm{mL}$. The CD4 or CD8 microbeads (Miltenyi Biotec) were used according to the manufacturer's instructions. Briefly, $10 \mu \mathrm{L}$ of CD4 or CD8a microbeads were added to $10^{7}$ splenocytes and incubated at $4^{\circ} \mathrm{C}$ for $15 \mathrm{~min}$. Cells were then washed and resuspended to $2 \times 10^{8}$ cells $/ \mathrm{mL}$. Splenocytes were separated using positive-selection MS columns. Finally, the $\mathrm{CD} 4^{+}$or $\mathrm{CD} 8^{+} \mathrm{T}$ cells were flushed out, and resuspended in X-vivo medium or complete PRIM 1640 medium for further TGF- $\beta 1$ production assay or Treg induction assay.

\section{T cell activation and cytokine production assays}

For in vivo assay of T cell activation, the isolated lung lymphocytes from naringenin treated mice or untreated surgical control mice were directly surface-stained with fluorochrome-conjugated anti-CD4, anti$\mathrm{CD} 8$, anti-CD44 and anti-CD62L antibodies. To analyze the in vivo production of IL-2 and IFN- $\gamma$, the isolated splenocytes from naringenin treated mice, untreated mice or normal naive mice were stimulated with $25 \mathrm{ng} / \mathrm{mL}$ phorbol 12-myristate 13-acetate (Sigma) and $500 \mathrm{ng} / \mathrm{mL}$ ionomycin (Sigma) for $5 \mathrm{~h}$. After further incubation with 
Brefeldin A Solution (eBioscience) for $3 \mathrm{~h}$, stimulated cells were surface stained with anti-CD8 antibodies, fixed and permeabilized in fixation/permeabilization buffer (eBioscience), and then stained with anti-IL-2 and anti-IFN-y antibodies (Chiang et al., 2007).

For the in vitro assay of $\mathrm{T}$ cell cytokine production, the isolated splenocytes or purified T cells from normal naive C57BL/6 mice were cultured with or without naringenin for $72 \mathrm{~h}$ in the presence of antiCD3 $(3 \mu \mathrm{g} / \mathrm{mL})$ and anti-CD28 $(1 \mu \mathrm{g} / \mathrm{mL})$ antibodies. The concentration of TGF- $\beta 1$ from purified T cells in the supernatant was determined by ELISA (Promega) according to the manufacturer's protocols. Cells were further stimulated, surface stained, fixed and permeabilized, and finally intracellularly stained for IL-10, as described above.

The antibody stained cells were analyzed by flow cytometry (FACSCalibur) and CellQuest software.

\section{Treg induction assay}

Isolated splenocytes of normal naive C57BL/6J mice were purified using CD4 MACS beads (Miltenyi Biotec, Germeny). The purified CD4 ${ }^{+} \mathrm{T}$ cells were cultured with anti-CD3 antibody $(3 \mu \mathrm{g} / \mathrm{mL})$, antiCD28 antibody $(1 \mu \mathrm{g} / \mathrm{mL})$ and TGF- $\beta 1(5 \mathrm{ng} / \mathrm{mL})$ to induce regulatory $T$ cells (Kong et al., 2009). Naringenin was supplemented to the culture at concentrations of 0 and $100 \mu \mathrm{mol} / \mathrm{L}$ for $72 \mathrm{~h}$. Cells treated with neither TGF- $\beta 1$ nor naringenin were used as normal controls. Cells were surfaced stained with anti-CD4 and anti-CD25 antibodies. To detect Foxp3 expression, the surface-stained cells were further intracellularly stained with anti-Foxp3 antibodies.

\section{Statistical analysis}

Data were presented as mean $\pm S D$. Statistical analyses were performed using the Student's $t$-test when comparing two groups. To compare three or more groups, ANOVA was performed with a post hoc Bonferroni test to determine which two groups showed significant differences. Survival curves were calculated by the Kaplan-Meier method and analyzed by the log rank test (GraphPad software, version 5.0 ). In all tests, $p<0.05$ was considered to be statistically significant.

\section{ACKNOWLEDGEMENTS}

This work was supported by The State Key Development Plan Project (Grant No. 2011CB707705).

\section{ABBREVIATIONS}

CTL, cytotoxic T lymphocytes; IFN- $\mathrm{y}$, interferon-gamma; IL-2, interleukin 2; IL-10, interleukin 10; LD50, medium lethal dose; MDSCs, myeloid-derived suppressor cells; MTT, 3-(4,5dimethylthiazol-2-yl)-2,5-diphenyltetrazolium bromide; Nar, naringenin; Tregs, regulatory T cells; TAMs, tumor-associated macrophages; TCR, T-cell antigen receptor; TGF- $\beta$, transforming growth factor- $\beta$

Supplementary material is available in the online version of this article at http://dx.doi.org/10.1007/s13238-011-1056-8 and is accessible for authorized authors.

\section{REFERENCES}

Ben-Eliyahu, S. (2003). The promotion of tumor metastasis by surgery and stress: immunological basis and implications for psychoneuroimmunology. Brain Behav Immun 17, S27-S36.

Beyer, M., and Schultze, J.L. (2006). Regulatory T cells in cancer. Blood 108, 804-811.

Bindea, G., Mlecnik, B., Fridman, W.H., Pages, F., and Galon, J. (2010). Natural immunity to cancer in humans. Curr Opin Immunol 22, 215-222.

Boomsma, M.F., Garssen, B., Slot, E., Berbee, M., Berkhof, J., Meezenbroek Ede, J., Slieker, W., Visser, A., Meijer, S., and Beelen, R.H. (2010). Breast cancer surgery-induced immunomodulation. J Surg Oncol 102, 640-648.

Brandacher, G., Winkler, C., Schroecksnadel, K., Margreiter, R., and Fuchs, D. (2006). Antitumoral activity of interferon-gamma involved in impaired immune function in cancer patients. Curr Drug Metab 7, 599-612.

Brune, I.B., Wilke, W., Hensler, T., Holzmann, B., and Siewert, J.R. (1999). Downregulation of T helper type 1 immune response and altered pro-inflammatory and anti-inflammatory $T$ cell cytokine balance following conventional but not laparoscopic surgery. Am J Surg 177, 55-60.

Chen, L., Huang, T.G., Meseck, M., Mandeli, J., Fallon, J., and Woo, S.L. (2007). Rejection of metastatic 4T1 breast cancer by attenuation of Treg cells in combination with immune stimulation. Mol Ther 15, 2194-2202.

Chen, W.J., and Konkel, J.E. (2010). TGF-beta and 'Adaptive' Foxp3(+) Regulatory T cells. J Mol Cell Biol 2, 30-36.

Chiang, J.Y., Jang, I.K., Hodes, R., and Gu, H. (2007). Ablation of Cblb provides protection against transplanted and spontaneous tumors. J Clin Invest 117, 1029-1036.

Coffelt, S.B., Hughes, R., and Lewis, C.E. (2009). Tumor-associated macrophages: effectors of angiogenesis and tumor progression. Biochim Biophys Acta 1796, 11-18.

Coughlin, S.S., and Ekwueme, D.U. (2009). Breast cancer as a global health concern. Cancer Epidemiol 33, 315-318.

Du, G., Jin, L., Han, X., Song, Z., Zhang, H., and Liang, W. (2009). Naringenin: a potential immunomodulator for inhibiting lung fibrosis and metastasis. Cancer Res 69, 3205-3212.

duPre, S.A., Redelman, D., and Hunter, K.W. Jr. (2008). Microenvironment of the murine mammary carcinoma 4T1: endogenous IFNgamma affects tumor phenotype, growth, and metastasis. Exp Mol Pathol 85, 174-188.

Faist, E., Kupper, T.S., Baker, C.C., Chaudry, I.H., Dwyer, J., and Baue, A.E. (1986). Depression of Cellular-Immunity after Major Injury - Its Association with Posttraumatic Complications and Its Reversal with Immunomodulation. Arch Surg-Chicago 121, 1000-1005.

Fang, F., Tang, Y., Gao, Z., and Xu, Q. (2010). A novel regulatory mechanism of naringenin through inhibition of $T$ lymphocyte function in contact hypersensitivity suppression. Biochem Biophys Res Commun 397, 163-169.

Gabrilovich, D.I., and Nagaraj, S. (2009). Myeloid-derived suppressor cells as regulators of the immune system. Nat Rev Immunol 9, 162-174.

Hogan, B.V., Peter, M.B., Shenoy, H.G., Horgan, K., and Hughes, T.A. (2011). Surgery induced immunosuppression. Surg-J R Coll Surg E 9, 38-43.

James, M.A., Lu, Y., Liu, Y., Vikis, H.G., and You, M. (2009). RGS17, an overexpressed gene in human lung and prostate cancer, 
induces tumor cell proliferation through the cyclic AMP-PKA-CREB pathway. Cancer Res 69, 2108-2116.

Jemal, A., Siegel, R., Xu, J., and Ward, E. (2010). Cancer statistics, 2010. CA Cancer J Clin 60, 277-300.

Kanno, S., Tomizawa, A., Hiura, T., Osanai, Y., Shouji, A., Ujibe, M., Ohtake, T., Kimura, K., and Ishikawa, M. (2005). Inhibitory effects of naringenin on tumor growth in human cancer cell lines and sarcoma S-180-implanted mice. Biol Pharm Bull 28, 527-530.

Ko, K., Yamazaki, S., Nakamura, K., Nishioka, T., Hirota, K., Yamaguchi, T., Shimizu, J., Nomura, T., Chiba, T., and Sakaguchi, S. (2005). Treatment of advanced tumors with agonistic anti-GITR $\mathrm{mAb}$ and its effects on tumor-infiltrating Foxp3 + CD25 + CD4 + regulatory T cells. J Exp Med 202, 885-891.

Kong, L.Y., Wei, J., Sharma, A.K., Barr, J., Abou-Ghazal, M.K., Fokt, I., Weinberg, J., Rao, G., Grimm, E., Priebe, W., et al. (2009). A novel phosphorylated STAT3 inhibitor enhances T cell cytotoxicity against melanoma through inhibition of regulatory $T$ cells. Cancer Immunol Immunother 58, 1023-1032.

Le Marchand, L., Murphy, S.P., Hankin, J.H., Wilkens, L.R., and Kolonel, L.N. (2000). Intake of flavonoids and lung cancer. J Natl Cancer Inst 92, 154-160.

Liyanage, U.K., Moore, T.T., Joo, H.G., Tanaka, Y., Herrmann, V., Doherty, G., Drebin, J.A., Strasberg, S.M., Eberlein, T.J., Goedegebuure, P.S., et al. (2002). Prevalence of regulatory T cells is increased in peripheral blood and tumor microenvironment of patients with pancreas or breast adenocarcinoma. J Immunol 169, 2756-2761.

Lohr, F., Hu, K., Haroon, Z., Samulski, T.V., Huang, Q., Beaty, J., Dewhirst, M.W., and Li, C.Y. (2000). Combination treatment of murine tumors by adenovirus-mediated local B7/IL12 immunotherapy and radiotherapy. Mol Ther 2, 195-203.

Marigo, I., Dolcetti, L., Serafini, P., Zanovello, P., and Bronte, V. (2008). Tumor-induced tolerance and immune suppression by myeloid derived suppressor cells. Immunol Rev 222, 162-179.

Markiewicz, D.A., Schultz, D.J., Haas, J.A., Harris, E.E., Fox, K.R., Glick, J.H., and Solin, L.J. (1996). The effects of sequence and type of chemotherapy and radiation therapy on cosmesis and complications after breast conservation therapy. Int J Radiat Oncol Biol Phys 35, 661-668.

McCahill, L.E., Privette, A., James, T., Sheehey-Jones, J., Ratliff, J., Majercik, D., Krag, D.N., Stanley, M., and Harlow, S. (2009). Quality measures for breast cancer surgery: initial validation of feasibility and assessment of variation among surgeons. Arch Surg 144, 455-462; discussion 462-463.

Ogawa, K., Hirai, M., Katsube, T., Murayama, H., Hamaguchi, K., Shimakawa, T., Naritake, Y., Hosokawa, T., and Kajiwara, T. (2000). Suppression of cellular immunity by surgical stress. Surgery $127,329-336$.
Ortiz-Andrade, R.R., Sanchez-Salgado, J.C., Navarrete-Vazquez, G., Webster, S.P., Binnie, M., Garcia-Jimenez, S., Leon-Rivera, I., Cigarroa-Vazquez, P., Villalobos-Molina, R., and Estrada-Soto, S. (2008). Antidiabetic and toxicological evaluations of naringenin in normoglycaemic and NIDDM rat models and its implications on extra-pancreatic glucose regulation. Diabetes Obes Metab 10, 1097-1104.

Page, G.G., and Ben-Eliyahu, S. (1997). Increased surgery-induced metastasis and suppressed natural killer cell activity during proestrus/estrus in rats. Breast Cancer Res Treat 45, 159-167.

Pulaski, B.A., and Ostrand-Rosenberg, S. (2001). Mouse 4T1 breast tumor model. Curr Protoc Immunol Chapter 20, Unit 20.2.

Pulaski, B.A., Terman, D.S., Khan, S., Muller, E., and OstrandRosenberg, S. (2000). Cooperativity of Staphylococcal aureus enterotoxin B superantigen, major histocompatibility complex class $\mathrm{II}$, and CD80 for immunotherapy of advanced spontaneous metastases in a clinically relevant postoperative mouse breast cancer model. Cancer Res 60, 2710-2715.

Rosenberg, S.A. (2001). Progress in human tumour immunology and immunotherapy. Nature 411, 380-384.

Salo, M. (1992). Effects of anaesthesia and surgery on the immune response. Acta Anaesthesiol Scand 36, 201-220.

Shafir, M., Bekesi, J.G., Papatestas, A., Slater, G., and Aufses, A.H. (1980). Preoperative and Postoperative Immunological Evaluation of Patients with Colorectal-Cancer. Cancer 46, 700-705.

Shenkier, T., Weir, L., Levine, M., Olivotto, I., Whelan, T., and Reyno, L. (2004). Clinical practice guidelines for the care and treatment of breast cancer: 15 . Treatment for women with stage III or locally advanced breast cancer. CMAJ 170, 983-994.

So, F.V., Guthrie, N., Chambers, A.F., Moussa, M., and Carroll, K.K. (1996). Inhibition of human breast cancer cell proliferation and delay of mammary tumorigenesis by flavonoids and citrus juices. Nutr Cancer 26, 167-181.

Townsend, S.E., and Allison, J.P. (1993). Tumor rejection after direct costimulation of CD8+ T cells by B7-transfected melanoma cells. Science 259, 368-370.

van der Bij, G.J., Oosterling, S.J., Bogels, M., Bhoelan, F., Fluitsma, D.M., Beelen, R.H., Meijer, S., and van Egmond, M. (2008). Blocking alpha2 integrins on rat CC531s colon carcinoma cells prevents operation-induced augmentation of liver metastases outgrowth. Hepatology 47, 532-543.

Whiteside, T.L. (2006). Immune suppression in cancer: effects on immune cells, mechanisms and future therapeutic intervention. Semin Cancer Biol 16, 3-15.

Yang, Y., Xu, Y., Xia, T., Chen, F., Zhang, C., Liang, W., Lai, L., and Fang, X. (2011). A single-molecule study of the inhibition effect of Naringenin on transforming growth factor-beta ligand-receptor binding. Chem Commun (Camb) 47, 5440-5442. 